\title{
ANL/IPNS/CP-103674
}

\section{Structure of Charged Polymer Chains in Confined Geometry}

\section{Elliot P. Gilbert ${ }^{1}$, Loïc Auvray ${ }^{2}$ and Jyotsana Lal ${ }^{1}$}

${ }^{1}$ Intense Pulsed Neutron Source, Argonne National Laboratory, Argonne, IL 60439, U.S.A.

${ }^{2}$ Laboratoire Leon Brillouin, Centre d'Etudes de Saclay, Gif-sur-Yvette Cedex, France.

\section{ABSTRACT}

The intra- and interchain structure of sodium poly(styrenesulphonate) when free and when confined in contrast matched porous Vycor has been investigated by SANS. When confined, a peak is observed whose intensity increases with molecular weight and the 1/q scattering region is extended compared to the bulk. We infer that the chains are sufficiently extended, under the influence of confinement, to highlight the large scale disordered structure of Vycor. The asymptotic behavior of the observed interchain structure factor is $\approx 1 / \mathrm{q}^{2}$ and $\approx 1 / \mathrm{q}$ for free and confined chains respectively.

\section{INTRODUCTION}

An understanding of polymer conformation in reduced dimensionality has enormous technological application, such as microfabrication and miniaturization [1]. While neutron scattering has provided invaluable information of chain conformation in bulk [2], there have been relatively few scattering experiments of chains under confinement and these mainly focus on neutral chains. The significantly more complex, and biologically relevant, situation of charged chains (e.g. DNA) in confined geometry currently lacks an experimental framework with which to verify fundamental principles. We report here small-angle neutron scattering (SANS) studies of poly(styrenesulphonate) (PSSNa) when free and when confined in the nanopores of Vycor. By employing solutions composed of either pure deuterated PSSNa or suitable mixtures of protonated and deuterated PSSNa, we have separated the intramolecular and intermolecular contributions to the total scattering, and provide a theoretical understanding of their q-dependencies.

\section{EXPERIMENTAL DETAILS}

All polyelectrolytes had a degree of sulphonation $>95 \%$ and were dialysed prior to solution preparation; molecular weights, $M_{W}$ and polydispersities, $M_{w} / M_{N}$, are shown in table 1 . Since Vycor is highly susceptible to contamination, disks were cleaned by repeated boiling in $\mathrm{H}_{2} \mathrm{O}_{2}$ solution followed by heating to $500^{\circ} \mathrm{C}$ for at least three hours. The cleaned disks were opalescent but, on entering the solution, became translucent with bubbles subsequently being released, indicating the solution-displacement of air. The confined samples were studied after 2,7 and 9 days for the low, intermediate and high $M_{W}$ samples respectively corresponding to the time for which no visible bubbles remained. Experiments were performed on the SAD instrument at IPNS, Argonne National Laboratory. Absolute values of intensity were obtained from calibration against a polymer standard. 


\section{DISCLAIMER}

This report was prepared as an account of work sponsored by an agency of the United States Government. Neither the United States Government nor any agency thereof, nor any of their employees, make any warranty, express or implied, or assumes any legal liability or responsibility for the accuracy, completeness, or usefulness of any information, apparatus, product, or process disclosed, or represents that its use would not infringe privately owned rights. Reference herein to any specific commercial product, process, or service by trade name, trademark, manufacturer, or otherwise does not necessarily constitute or imply its endorsement, recommendation, or favoring by the United States Government or any agency thereof. The views and opinions of authors expressed herein do not necessarily state or reflect those of the United States Government or any agency thereof. 


\section{DISCLAIMER}

Portions of this document may be illegible in electronic image products. Images are produced from the best available original document. 


\section{REGEIVED}

\section{JAN 052001}

Table I. Polyelectrolytes studied, $\mathrm{R}_{\mathrm{g}}$ and Sharp and Bloomfreldinodelfitting parameters for bulk ZAC solutions. $\mathrm{R}_{\mathrm{g}}$ limits given for polystyrene, rod conformation and Benoit and Doty relation

\begin{tabular}{|c|c|c|c|c|c|c|}
\hline $\mathrm{M}_{\mathrm{W}}(\mathrm{Da}) \mathrm{H} / \mathrm{D}$ & $31000 \mathrm{H}$ & $25000 \mathrm{D}$ & $76000 \mathrm{H}$ & $77400 \mathrm{D}$ & $451000 \mathrm{H}$ & $425000 \mathrm{D}$ \\
\hline $\mathrm{M}_{\mathrm{w}} / \mathrm{M}_{\mathrm{N}}$ & 1.1 & 1.1 & $<1.1$ & 1.1 & 1.05 & 1.04 \\
\hline$\langle\mathrm{N}\rangle_{\mathrm{ZAC}}$ & \multicolumn{2}{|c|}{142} & \multicolumn{2}{|c|}{368} & \multicolumn{2}{|c|}{2142} \\
\hline$\left\langle\mathrm{M}_{\mathrm{W}}\right\rangle_{\mathrm{ZAC}}$ & \multicolumn{2}{|c|}{29500} & \multicolumn{2}{|c|}{76300} & \multicolumn{2}{|c|}{445000} \\
\hline $\mathrm{R}_{\mathrm{g}}$ (neutral) & \multicolumn{2}{|c|}{$36 \AA$} & \multicolumn{2}{|c|}{$58 \AA$} & & $143 \mathrm{~A}$ \\
\hline $\mathrm{R}_{\mathrm{g}}$ (rod) & & & & & & \\
\hline $\mathrm{R}_{\mathrm{g}}$ (BD) & \multicolumn{2}{|c|}{$40 \AA$} & \multicolumn{2}{|c|}{$70 \AA$} & \multicolumn{2}{|c|}{$178 \AA$} \\
\hline Parameter & Fit & Pred. & Fit & Pred. & Fit & Pred. \\
\hline $1_{p}(\AA)$ & $15(1)$ & & $17(1)$ & & $18(1)$ & \\
\hline Scale factor & $0.90(2)$ & 1.20 & $1.8(1)$ & 2.42 & $16.2(8)$ & 18.1 \\
\hline
\end{tabular}

The SANS from bare Vycor exhibits an intense peak at $q_{\max }$ ca. $0.02 \AA^{-1}$ arising from quasiperiodicity of the, average internal diameter of $70 \AA$, pore structure [3]. The scattering length density (SLD) of Vycor was determined by contrast variation experiments with mixtures of Millipore $\mathrm{H}_{2} \mathrm{O}$ (resistivity equal to $18 \mathrm{M \Omega cm}$ ) and $\mathrm{D}_{2} \mathrm{O}$. The match point was determined to be $3.79 \times 10^{10} \mathrm{~cm}^{-2}$; equivalent in SLD to a mixture of $37.6 \% \mathrm{H}_{2} \mathrm{O}$ and $62.4 \% \mathrm{D}_{2} \mathrm{O}$. To confirm this, Vycor was filled with such a water mixture and gave rise to a SANS signal in which the correlation peak is absent and the scattered intensity at $\mathrm{q}_{\max }$ was reduced by three orders of magnitude. This water composition was used to prepare all polyelectrolyte solutions. The same Vycor disk was used for both the sample and background scattering runs to avoid effects from differences between individual disks.

\section{THEORY}

The scattering from a deuterated/ protonated mixture of a single polymer species, with index of polymerisation, $N$, in solution may be written [4]:

$$
I(q)=\left(\bar{\rho}_{D}-\bar{\rho}_{H}\right)^{2} x(1-x) v \Phi N P(q)+\left(x \bar{\rho}_{D}+(1-x) \bar{\rho}_{H}-\bar{\rho}_{0}\right)^{2}\left[v \Phi N P(q)+V \Phi^{2} Q(q)\right]
$$

where I $(q)$ is the total scattering intensity per unit volume $\left(\mathrm{cm}^{-1}\right), \bar{\rho}_{D}, \bar{\rho}_{H}, \bar{\rho}_{0}$ are the SLD of the deuterated, protonated polymer, solvent respectively, $x$ is the mole fraction of deuterated chains, $v$ is the monomer molecular volume, $V$ is the sample volume and $\Phi$ is the total volume fraction of polymer in solution. $P(q)$ and $Q(q)$ are the (intrachain) form factor and interchain structure factors respectively as normalised in [4]. When the average contrast between the polymer and solvent is adjusted to zero (ZAC, zero average contrast condition), the scattered intensity becomes:

$$
I(q)=\left(\bar{\rho}_{D}-\bar{\rho}_{H}\right)^{2} x(1-x) \nu \Phi N P(q)
$$

enabling direct determination of $\mathrm{P}(\mathrm{q})$. To maximise the scattering intensity, the composition of the polymer mixture is typically chosen with $x$ equal to 0.5 with the solvent matched accordingly 
but, for confined studies, both the solute and solvent must match Vycor. With SLDs for PSS ${ }^{\mathrm{N}} \mathrm{Na}$ and $\mathrm{PSS}^{\mathrm{H}} \mathrm{Na}$ of 6.8879 and $2.8321 \times 10^{-10} \mathrm{~cm}^{-2}$ respectively [5], this is satisfied for a mixture of 23.6:76.4 mole\% but necessarily results in a further reduction in scattering intensity in these already weakly scattering systems.

For a medium with neutral pores, a concentration is typically chosen such that the Debye length, $\kappa^{-1}$, is less than the pore diameter. Initial experiments were performed with solutions with $\kappa^{-1} \approx 7 \AA$ but resulted in negligible chain penetration indicating the charged nature of the pore surface. The concentration used for all ZAC solutions here was $0.306 \mathrm{gcm}^{-3}$ (ca. four times the initial study) except for the intermediate weight solution which was $0.238 \mathrm{gcm}^{-3}$. Similar deuterated-only solutions were also prepared for the low and high $\mathrm{M}_{\mathrm{W}}$ chains with a concentration of $0.314 \mathrm{gcm}^{-3}$ so as to maintain a constant monomer concentration.

The radius of gyration, $R_{g}$, for a fully extended chain is $L / \sqrt{ } 12$, where $L$ is the contour length (equal to $\mathrm{Na}$ where a is the monomer size equal to $2.5 \AA$ ). Using this value to calculate the overlap concentration, $\mathrm{c}^{*}$, for the ZAC solutions gives $4.52 \times 10^{-2}, 6.79 \times 10^{-3}$ and $2.00 \times 10^{-4}$ $\mathrm{gcm}^{-3}$ respectively where:

$$
c^{*}=M_{\mathrm{w}} / \mathrm{N}_{\mathrm{A}} \mathrm{R}_{\mathrm{g}}{ }^{3}
$$

and $\mathrm{N}_{\mathrm{A}}$ is Avogadro's number. For comparison, $\mathrm{R}_{\mathrm{g}}$ for the neutral parent polystyrene chain in a theta solvent is $0.27 \mathrm{M}_{\mathrm{w}}{ }^{0.506}[6] . \mathrm{R}_{\mathrm{g}}$ limits for the chains studied are summarised in Table $\mathrm{I}$.

\section{RESULTS AND DISCUSSION}

The scattering from the bulk deuterated chain is weak due to the low compressibility of the system. The principal feature is a peak at q ca. $0.25 \AA^{-1}$ associated with an average interchain distance of ca. $25 \AA$ whose position is independent of molecular weight. From the bulk ZAC solutions, we are sensitive only to the chain form factor. Kratky plots, I(q) versus $\mathrm{q}^{-2}$, exhibit a plateau region centred at $q$ ca. $0.05 \AA^{-1}$ and, at higher $q$, vary as $q^{-1}$. The scattering vector associated with this crossover, $\mathrm{q}^{*}$, corresponds to the transition between the asymptotic behaviour of a Gaussian chain to that of a rod. The persistence length, $1_{\mathrm{p}}$, can thus be extracted from fitting the scattering to a Sharp and Bloomfield model for wormlike chains if $L>10 \mathrm{I}_{\mathrm{p}}$ and $\mathrm{ql}_{\mathrm{p}}<2$ (Figure 1)[7]. Setting L equal to 356, 919 and $5356 \AA \AA$ for the low, intermediate and high $\mathrm{M}_{\mathrm{w}}$ chains based on their average degree of polymerisation, $\langle\mathrm{N}\rangle_{\mathrm{ZAC}}$, the parameters obtained are summarised in Table $I$. One may express $R_{g}$ as a function of $L$ and $l_{p}$ by [8]:

$$
\left\langle\mathrm{R}_{\mathrm{g}}^{2}\right\rangle=l_{\mathrm{p}}^{2}\left[\frac{\mathrm{L}}{3 l_{\mathrm{p}}}-1+\frac{2 l_{p}}{L}-\frac{2 l_{p}^{2}}{L^{2}}\right]\left(1-\exp \left(-\frac{L}{l_{p}}\right)\right)
$$

$R_{g}$ values obtained from (4) are much closer to those for the Gaussian relative to rod conformation as expected for the chain concentrations $» c^{*}$ investigated (Table I). $l_{p}$ is also independent of $\mathrm{M}_{\mathrm{W}}$ at this high monomer concentration. A comparison of the free and confined scattering at large scattering vectors $\left(\mathrm{ql}_{\mathrm{p}}>>1\right)$ indicates that the intensity associated with the confined chain is approximately one quarter that of the bulk solution. Since Vycor has a porosity of $28 \%$, such a reduction in high $q$ scattering is in line with expectations and suggests that chains 
have fully entered. The principal feature in the confined scattering is a peak at approximately the same position as observed from bare Vycor whose intensity increases with $\mathrm{M}_{\mathrm{w}}$. In previous studies of the neutral parent polymer under confinement, the SANS from chains of $\mathrm{M}_{\mathrm{W}} \geq 137$ $\mathrm{kDa}$ showed the slight presence of this peak despite being at ZAC [4]. This was interpreted in terms of chains spanning pores over a range comparable to or longer than the Vycor pore correlations. We may exclude the possibility that this peak arises from a contrast mismatch since not only has the contrast match point of Vycor been verified but the bulk ZAC scattering gives rise to $I(q), l_{p}$ and $R_{g}$ in good agreement with the existing literature [5]. Figure 1 shows scattering data plotted in the form of a $\mathrm{qP}(\mathrm{q})$ versus $\mathrm{q}$ form to emphasise the asymptotic rod-like behaviour and shows that this conformation region extends to much lower $q$ than the corresponding bulk indicating an increase in the persistence length under confinement. The low $q$ cut-off for $\mathrm{q}^{-1}$ scattering occurs at $q \mathrm{ca}$. $0.05 \AA^{-1}$, independent of $\mathrm{M}_{\mathrm{w}}$; below this $\mathrm{q}$, the scattering around the peak masks the polymer scattering. We also note that the observed increase in peak intensity with $\mathrm{M}_{\mathrm{W}}$ is as would be expected if an increasing number of pores were connected by an extended chain. In common with the ZAC chains, the confined deuterated polyelectrolytes exhibit a correlation peak with the higher $\mathrm{Mw}_{w}$ chain associated with a greater intensity [9]. The deuterated sample gives rise to lower scattering for fixed $\mathrm{M}_{\mathrm{W}}$ due to the presence of intermolecular effects which are absent under the ZAC condition [9].


Figure 1. $q P(q)$ from free and confined, zero average contrast polyelectrolytes for low and high $M_{W}$. Free chains have been fitted to the Sharp and Bloomfield model for $q l_{p}<2$. Also shown for high $M_{W}$ is the crossover region at $q^{*}$ between $q^{-2}$ and $q^{-1}$ scattering. 
There have been few studies in which $Q(q)$ has been measured for free polyelectrolyte chains and none in which a quantitative analysis of its variation with $\mathrm{q}$ has been discussed; however, the combination of ZAC and deuterated chain scattering ( $x=1$ in (1)) here enables the separation of intrachain, $P(q)$ and interchain contributions, $Q(q)$. Using the measured $P(q)$ from $Z A C$ and inserting the known values for the prefactors into (1), we may obtain $Q(q)$ by an appropriate weighted subtraction (Figure 2). One of the striking features of our data is that $Q(q)$ of the free and confined chains have well-defined asymptotic behaviour; $Q(q)$ decays as $\approx-q^{-2}$ for the bulk chains whereas the confined chain function decays only as $\approx-\mathrm{q}^{-1}$.
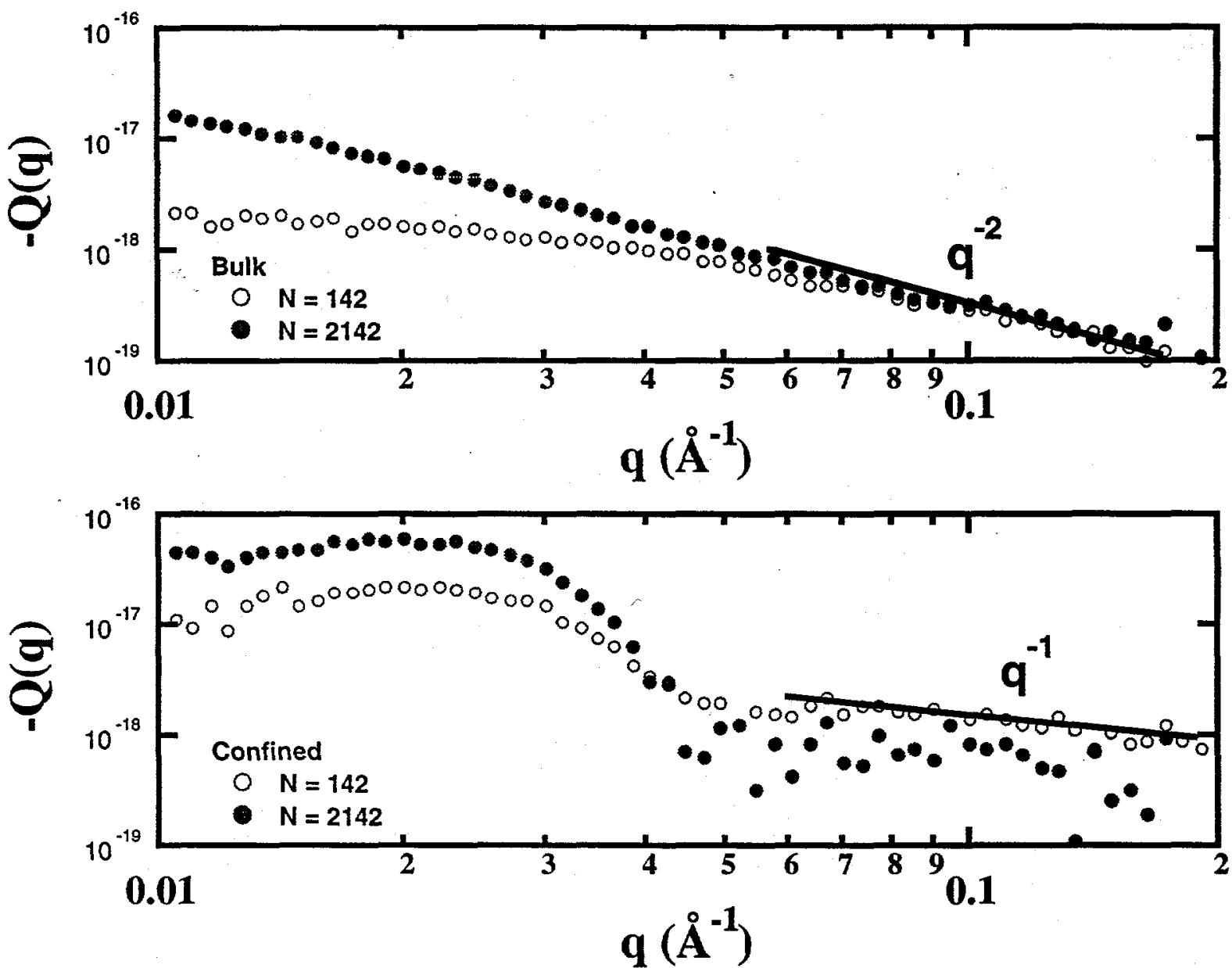

Figure 2. $-Q(q)$ for a) bulk and b) confined chains for low and high molecular weights.

We note that the effect of confined charged chains in water (dielectric constant $\approx 80$ ), surrounded by low dielectric constant $(=3.85)$ walls of Vycor glass would be to concentrate electric field lines along the axial direction of the pores [10]. This has the effect of enhancing the electrostatic interaction along the pore axis and may also be responsible for the stretched conformation as evidenced by the scattering peak. Further, since we are studying the semi-dilute regime, we may expect $Q(q)$ to be independent of $M_{w}$ in the bulk, at large $q$, where the scattering corresponds to dimensions $\approx l_{p}$ (Figure $2 a$ ). We thus believe that the observed behaviour is a simple consequence of the local rod-like structure of the chains. It has been suggested that, for 
bulk polyelectrolytes, $\mathrm{Q}(\mathrm{q})$ can be approximated by $\mathrm{q}^{-(2 v)}$ where $\mathrm{v}=1$ for a locally rigid rod in contrast to the experimentally verified $\mathrm{q}^{-3}$ decay for neutral chains [11]. This is indeed verified by our experimental data for the bulk systems (Figure 2a). It may also be easily shown the asymptotic limit of $\mathrm{Q}(\mathrm{q})$ for a solution of rod-like particles is $\pi^{2} / 4 \mathrm{q}^{2} \mathrm{~L}^{2}$ and is proportional to the amplitude scattered by a portion of rod of size $\approx q^{-1}[16]$. On the contrary, if we assume that the confined polyelectrolyte chains are locally aligned in the pores, as for a single rod, the main contribution to $Q(q)$ will vary as $q^{-1}[16]$. We find for the bulk that the $Q(q)$ functions not only overlay at high $q$ but also illustrate the predicted $q^{-2}$ power law. While statistics limit our conclusions at high $\mathrm{M}_{\mathrm{W}}$, in the case of the confined chains, the low $\mathrm{M}_{\mathrm{W}}$ system does indeed exhibit the $\mathrm{q}^{-1}$ behaviour as predicted for aligned rods.

\section{CONCLUSIONS}

SANS has been used to investigate the structure of PSSNa in a bulk concentrated solution and when confined in Vycor. The persistence length obtained from the Sharp and Bloomfield model for wormlike chains is independent of the molecular weight in the bulk solution. When confined in Vycor, and under the ZAC condition, a broad peak is present at the bare Vycor peak position whose intensity increases with molecular weight. We infer that the chains are sufficiently extended, under the influence of confinement, to highlight the large scale disordered structure of Vycor. The 1/q scattering region is extended compared to the bulk and supports this view. We have also determined $Q(q)$ for both the bulk and confined states. The asymptotic behavior of the observed interchain structure factor is $\approx 1 / \mathrm{q}^{2}$ for free and $\approx 1 / \mathrm{q}$ for confined chains.

\section{ACKNOWLEDGMENTS}

We would like to thank Dr. A. Lapp for preparation of the low $\mathrm{M}_{\mathrm{w}}$ chains and Mr. E. Lang for assistance in performing SANS studies. This work has benefited from the use of the Intense Pulsed Neutron Source at Argonne National Laboratory which is funded by the U.S. Department of Energy, BES-Materials Science, under Contract W-31-109-ENG-38.

\section{REFERENCES}

[1] S.H.J. Idziak and Y.L. Li, Current Opinion Colloid Interface Sci., 3, 293 (1998).

[2] P.-G. de Gennes in Scaling Concepts in Polymer Physics, (Cornell University Press, 1979).

[3] P. Levitz et al., J. Phys. Chem., 95, 6151 (1991).

[4] J. Lal, S.K. Sinha and L. Auvray, J. Phys. II (France), 71597 (1997).

[5] M.N. Spiteri et al., Phys. Rev. Lett., 77, 5218 (1996).

[6] J.-P. Cotton, J. Phys. Lett., 41, L231 (1980).

[7] P. Sharp and V.A. Bloomfield, Biopolymers, 6, 1201 (1968).

[8] H. Benoît and P. Doty, J. Phys. Chem., 57, 958 (1953).

[9] E.P. Gilbert, L. Auvray and J. Lal, submitted.

[10] A. Parsegian, Nature, 221, 844 (1969).

[11] G. Jannink et al., Europhys. Lett., 27, 47 (1994).

The submitted manuscript has been created by the University of Chicago as Operator of Argonne National Laboratory ("Argonne") under Contract No. W-31-109-ENG-38 with the U.S. Department of Energy. The U.S. Government retains for itself, and others acting on its behalf, a paid-up, nonexclusive. irrevocable worldwide license in said article to reproduce, prepare derivative works, distribute copies to the public, and perform pubiicly and display publicly, by or on behalf of the Government. 